\title{
Pour une approche interactive des savoirs locaux : l'ethno-éthologie
}

\section{Florence Brunois}

\section{(2) OpenEdition \\ 12 Journals}

Édition électronique

URL : http://journals.openedition.org/jso/335

DOI : $10.4000 /$ jso.335

ISSN : $1760-7256$

Éditeur

Société des océanistes

\section{Édition imprimée}

Date de publication : 1 décembre 2005

Pagination : $31-40$

ISSN : 0300-953x

\section{Référence électronique}

Florence Brunois, «Pour une approche interactive des savoirs locaux : l'ethno-éthologie », Journal de la Société des Océanistes [En ligne], 120-121 | Année 2005, mis en ligne le 27 novembre 2008, consulté le 01 mai 2019. URL : http://journals.openedition.org/jso/335 ; DOI : 10.4000/jso.335 


\section{Pour une approche interactive des savoirs locaux : l'ethno-éthologie}

par

Florence BRUNOIS*

\section{RÉSUMÉ}

Cet article présente une approche alternative des savoirs locaux: l'ethno-éthologie. Prônant le relativisme ontologique comme précepte, cette démarche recontextualise l'étude des modes de connaissance dans la complexité interactive liant les hommes au monde vivant afin d'isoler l'incidence qu'exercent les comportements spécifiques et interspecifiques des non-humains sur la constitution des savoirs.

MOTS-CLÉS : ethno-éthologie, savoirs locaux, interactivité, relativisme ontologique, biodiversité, catégorisation, Kasua.

Re-contextualiser l'étude des savoirs locaux dans la complexité interactive, idéelle et matérielle, liant les hommes au monde vivant, est l'ambition de l'approche ethnologique que je propose aujourd'huil. Conçue ainsi, cette démarche constitue une autre manière d'appréhender les modes de connaissances, une sorte d'entrée méthodologique qui, de par sa portée globalisante et dialectique, permet d'éclairer un aspect fondamental laissé dans l'ombre par des analyses menées à partir de perspectives trop

\section{ABSTRACT}

This article proposes an alternative way to study folk knowledge: the ethno-ethology. Recommending the ontological relativism as precept, this approach re-contextualizes the study of knowledge modalities in the interactive complexity linking humans and the living world. The aim is to determine the influence that non humans' specific and interspecific behaviors exert on the constitution of the Folk-knowledge and ecological know how.

KeYwORDS: Ethno-ethology ; folkknowledge, interactivity, ontological relativism, biodiversity, categorization, Kasua souvent anthropocentrées, celui de l'incidence qu'exercent les êtres vivants et leurs interactions avec les hommes sur la constitution des savoirs et savoir-faire locaux.

Issue de mon expérience de terrain auprès des Kasua de Papouasie Nouvelle-Guinée, cette approche « alternative » des modes de connaissances se présente ainsi comme une mise en application des résultats scientifiques de Peter Dwyer. Qu'ils portent sur les taxonomies, les connaissances zoologiques ou encore les concep-

1. Cette approche a été réfléchie au cours d'un financement post-doctoral de la Fondation Fyssen que je tiens ici à remercier. Je voudrais également remercier Claudine Friedberg pour la justesse des commentaires qu'elle a apporté à ce présent travail.

* uMR 5145 CNRS/MNHN/Paris vII, brunois@mnhn.fr 
tions des animaux, tous parviennent, nous l'avons vu, à une même conclusion : "ils émergent des interactions entre les hommes et le monde" (Dwyer, 1976: 433, cf. Supra p. 6). Reste que la mise en pratique d'un tel acquis scientifique n'est pas aussi aisé qu'il y paraît : elle suppose l'adoption d'une nouvelle approche des êtres vivants. Reconnaître le caractère interactif des relations hommes/êtres vivants implique en effet de repenser nécessairement le traitement que l'ethnoscience accordait jusqu'à présent à tous les «existants liés à l'homme " (Descola, 2001 : 19). Il n'est plus envisageable de les considérer comme de simples objets, passifs, convoqués sur la scène sociale pour nous entretenir dans un monologue humanocentrique. Une " désobjectivation " s'impose pour leur réattribuer leur pleine identité d'êtres vivants, c'est-àdire d'êtres capables d'agir et d'interagir sur le monde, notre monde. Dans ces circonstances, tant la démarche visant à appréhender les nonhumains que celle destinée à comprendre la perception et la conception qu'en ont les hommes ne peuvent plus se limiter à la seule étude de leurs qualités morphologiques. Elles doivent désormais intégrer de manière systématique l'analyse de leurs comportements spécifiques et interspécifiques tels qu'ils sont vécus par les sociétés étudiées et, simultanément, interroger l'incidence qu'ils exercent sur l'élaboration des connaissances, c'est-à-dire mener une ethno-éthologie ${ }^{2}$.

Nous le constatons, la redéfinition de notre objet d'étude entraîne simultanément celle de notre problématique. En d'autres termes, elle bouleverse considérablement la donne. Pourtant, nous allons découvrir qu'une telle reformulation s'impose aujourd'hui, d'un point de vue scientifique, tout d'abord si l'on considère les avancées les plus récentes des diverses disciplines abordant de près la question des modes de connaissances, ensuite et surtout, d'un point de vue ethnographique si l'on cherche à appréhender le savoir des autres en reconnaissant qu'ils sont autres.

\section{Quand les modes de connaissances résultent d'un engagement inter-relationnel}

Recontextualiser l'étude des savoirs locaux dans l'interactivité liant les hommes au monde vivant inscrit notre nouvelle approche au cœur de la réflexion qui anime aujourd'hui l'anthropologie de la nature, la sociologie des sciences et les sciences cognitives, dont l'objet est précisément de rendre compte du caractère intégré des modes de connaissances. L'enjeu de cette réflexion est d'ailleurs immense puisqu'il s'agit d'une remise en cause profonde des concepts de « nature » et de « culture » et, surtout, du bien fondé épistémologique de leur dualité paradigmatique dans la constitution et l'appréhension des savoirs. Certes, la question n'est pas foncièrement nouvelle! Depuis les travaux pionniers d'André G. Haudricourt (1962, 1964), nombreuses sont les recherches en ethnoscience qui ont montré l'impossibilité d'appréhender les connaissances de l'environnement indépendamment des relations que les individus entretiennent avec ses composantes ou encore indépendamment des relations que les individus entretiennent entre eux. En effet, si les sociétés étudiées reconnaissent généralement les discontinuités objectives rencontrées dans le monde vivant, les structures de leur savoir - c'est-à-dire l'organisation de leurs connaissances - et leur mise en pratique ne traduisent pas un ordre naturel qui serait indépendant de leur culture - voir entre autres: Conklin (1962), Bulmer, 1967, 1968), Dwyer (1976, 1984-1985, 1996), Barrau (1967, 1971, 1975), Grenand (1980), Bahuchet (1989), Chaumeil (1983), Friedberg (1990, 1997), Revel (1990), Ellen (1993), Minnegal (1996), Hornborg (1996), Hviding (1996), Howell (1996) et Arhem (1996). Les savoirs locaux se présentent comme des savoirs « culturellement » intégrés : ils transgressent le dualisme. L'anthropologie sociale rencontrera un phénomène similaire. En 1980, Marylin Strathern explique ainsi combien pour la société d'Hagen (Hautes Terres de la Papouasie Nouvelle-Guinée) :

« la nature et la culture ne sont pas des concepts qui ordonnent; la tension entre les termes est différente. En fait, on ne peut attribuer une signification unique à la nature ou à la culture comme le fait la pensée occidentale. Il n'existe pas (chez les Hagener) une dichotomie consistante, juste une matrice de contraste. » (1980:177)

En 1986, dans la première monographie consacrée à cette question épineuse, Philippe Descola précise les tenants de la conception originale des Ashuar d'Amazonie pour qui :

2. Dans les Mythologiques, Claude Lévi-Strauss nous avait déjà sensibilisé à l'importance de considérer les comportements de la faune et de la flore, mais il s'agissait d'un travail de cabinet nourri d'informations de zoologues et de botanistes et non d'observations in situ dans les sociétés où les comportements sont observés. Une démarche qui se différenciait de celle pratiquée et enseignée par André G. Haudricourt qui insistait sur l'aspect « concret » des relations liant l'homme à son environnement ( $c f$. infra p. 38). Cette différence n'empêchait toutefois pas André G. Haudricourt d'assister régulièrement à l'enseignement que délivrait Lévi-Strauss au Collège de France. 
« le champ culturel est singulièrement englobant, puisque s'y trouvent rangés des animaux, des plantes et des esprits qui ressortissent au domaine de la nature dans d'autres sociétés amérindiennes. On ne trouve donc pas chez les Ashuar cette antinomie entre deux mondes clos et irréductiblement opposés : le monde culturel de la société humaine et le monde naturel de la société animale. » (1986 : 399)

D'autres cas ethnographiques vinrent s'ajouter à ces démonstrations témoignant de l'existence d'une pluralité des conceptualisations du monde vivant. Cependant, cet acquis anthropologique n'eut pas l'impact épistémologique escompté : il ne favorisa pas une reconsidération de l'approche ethnologique des savoirs locaux. Dominée peut-être par le "réalisme cognitif » prôné par les anglosaxons, l'ethnoscience persista à chercher comment les connaissances des autres intègrent deux domaines a priori dissociés, la culture et/ou la nature.

\section{Un tournant obligé dans l'histoire de l'anthropo- logie et de l'ethnoscience}

En fait, et comme il est souvent d'usage dans l'histoire des idées, la question gagna en pertinence à la suite d'analyses relevant, non pas de l'anthropologie, mais de la sociologie des sciences. À l'appui de données ethnographiques recueillies au sein des laboratoires où se produisent les expériences et la connaissance scientifique, Bruno Latour va démontrer en effet combien la science, bien qu'elle « s'arrache justement à tout contexte, à toute trace de contamination idéologique et sociale » $(1991: 126)$, transgresse elle aussi l'opposition binaire: elle crée des 《 quasi-objets » « quasi sujets », c'est-à-dire des hybrides relevant à la fois de la nature et de la culture - voir Bruno Latour $(1979,1991)$ et, plus récemment, Detlev Nothnagel (1993). Bref, à l'image des sociétés papoues ou amazoniennes auxquelles Bruno Latour fait d'ailleurs référence, les scientifiques ne séparent pas plus les humains des non-humains. Cette démonstration relativisait farouchement la prétendue modernité des occidentaux! Elle éprouvait précisément le "Grand Partage » constitutif de cette modernité, c'est-à-dire « le Grand Partage extérieur » entre Nous (les Occidentaux) et Eux (tous les autres), se fondant et s'expliquant par l'autre " Grand Partage intérieur », celui établi entre les humains et les non-humains, la science et la société ; un « Grand Partage intérieur » que ne reconnaissent pas les « autres» :

« incapables de séparer vraiment ce qui est connaissance de ce qui est société, ce qui est signe de ce qui est chose, ce qui vient de la nature telle qu'elle est de ce que requièrent leurs cultures. Quoi qu'ils fassent [...], ils [les autres] restent toujours aveuglés par cette confusion. » (Latour, 1991 : 135)

Développée, entre autres, dans son livre Nous n'avons jamais été modernes, son analyse provoqua de vives réactions dans le cénacle scientifique. Dans L'invention des sciences modernes, Isabelle Stengers ne manque pas de signaler que :

« une rumeur inquiétante se propage dans le monde des scientifiques. Il existe, paraît-il, des chercheurs, spécialistes des sciences humaines qui plus est, qui s'en prennent à l'idéal d'une science pure [...]. Ce champ mettrait en question toute séparation entre les sciences et les sociétés. » (1995: 11)

Et, effectivement, si l'on considère avec Philippe Descola (1996 : 98) que « supprimer l'idée de nature et tout l'édifice philosophique des réalisations occidentales s'effondrera ", la crainte exprimée par cette philosophe des sciences se justifie. En revanche, l'inquiétude perd soudainement de sa portée si l'on envisage la question d'un point de vue anthropologique. À la différence des philosophes ou des épistémologues, les ethnologues, comme je le soulignais, ont depuis longtemps recensé, appréhendé et interprété des systèmes de connaissances complexes qui ne reconnaissent pas une nature universelle distincte de la culture. C'est pourquoi l'anthropologie est à même de relativiser ce "cataclysme intellectuel », cette éventuelle "révolution kuhniennne " et de considérer que les effets vont seulement « reconfigurer notre cosmologie et la rendre moins exotique pour les nombreuses cultures qui sont prêtes à embrasser les valeurs de celles qu'elles croient relever de la modernité » (Descola, 1996 : 98). Davantage encore, l'exercice auquel s'est prêtée la sociologie des sciences et dont les résultats ont discrédité - c'est-à-dire relativisé - l'altérité culturelle reposant sur le grand partage entre nature et culture vient enrichir l'anthropologie. Ouvrant vers un " relativisme ontologique » dans lequel s'élaborent les connaissances, cette analyse permet à notre discipline de prendre enfin position dans ce débat qu'elle a de fait initié et alimenté. Celle-ci sera en effet consacrée dans la publication simultanée en 1996 de deux ouvrages majeurs : Redefining Nature: Ecology, Culture and Domestication (Roy Ellen et Kiyoshi Fukui [eds]) et Nature and Society. Anthropological Perspectives (Philippe Descola et Gisli Palsson [eds]). Foisonnant d'exemples ethnographiques venant tous contredire l'universalité de la dichotomie nature/ culture et donc sa valeur comme outil intellectuel pour appréhender les autres et leurs savoirs, 
l'ensemble des spécialistes réunis parviennent à une conclusion aux prétentions théoriques unanimes : la nécessité d'abandonner le traditionnel paradigme dualiste, certes " pilier fondateur de l'épistémologie moderne » (Descola et Palsson, 1996 : 12), mais dont la catégorie "nature» apparaît bien comme " une invention, un artifice de la pensée occidentale » (Dwyer, 1996 : 157), autrement dit, reconnue comme pouvant exercer un véritable obstacle épistémologique à la compréhension des "autres" et des savoirs des autres qui « sont des "autres" parce que précisément, ils ont d'autres "autres" " (Viveiros de Castro,1996 : 80).

\section{Vers de nouvelles perspectives de recherche}

Ces deux ouvrages traduisent un tournant considérable et obligé dans l'histoire de l'anthropologie et, bien sûr, dans l'histoire de l'ethnoscience. Logiquement, repenser l'interface nature/société signifie repenser simultanément l'approche des savoirs des autres. Chacun se l'accorde : en supprimant l'idée d'une nature séparée de la culture, notre discipline fait face à « un paysage intellectuel complètement différent, où l'état et les substances sont remplacés par les processus et les relations » (Descola et Palsson, 1996 : 12). Ce qui implique de reformuler à la fois notre objet et sa problématique. Il ne s'agit plus de relever simplement les usages des animaux et des plantes, les noms attribués aux espèces vivantes comme si ces connaissances et ces êtres participaient d'un corpus autonome et objectivé, ou encore d'inventorier les seules «bizarreries» culturelles que les savoirs des autres recèleraient. Adopter ces perspectives persisterait en effet à réitérer la dichotomie société/environnement et donc à supposer aux savoirs locaux une vision naturaliste, bref à se prêter à un péché d'occidocentrisme. Non, notre recherche doit désormais «situer l'action et l'intention dans le contexte d'un engagement mutuel entre les gens et l'environnement» (Ingold, 1996) et « examiner les savoirs liés aux plantes et aux animaux dans le cadre de l'ensemble de ces relations » (Friedberg, 1997). La perspective est certainement innovante. Reste que le plus important à considérer est qu'elle n'est pas marginale. Elle est partagée par les sciences cognitives qui constatent elles aussi que «la connaissance n'assume pas une intériorisation de l'environnement mais un comportement efficace dans un contexte donné » (Maturana et Varela, 1987: 172); qu'elle est « une relation négociée avec la nature » (Bird, 1987), ou encore, que le processus d'apprentissage est « une éducation de l'attention » (Gibson, $1979: 254$ ), soit un dialogue entre l'individu, la société et l'environnement s'inscrivant dans « une communauté de pratiques interactives dans lesquelles s'engage pleinement l'individu » (Lave, 1993).

Ainsi, quelle que soit la discipline choisie l'anthropologie, les sciences cognitives ou la sociologie des sciences -, le nouveau champ d'investigation est clairement similaire : comprendre les modes des connaissances des uns et des autres, c'est comprendre les processus interrelationnels dans lesquels les individus s'engagent avec les humains et les non-humains participant de leur monde. La question des modes de connaissances se pose donc aujourd'hui dans un contexte foncièrement nouveau et complexifié qui recquiert l'élaboration d'une nouvelle approche des savoirs ${ }^{3}$ et du monde vivant qu'ils appréhendent. Mon ambition est précisément de proposer une démarche affirmant clairement ce renouveau. Euvrant pour une écologie authentique des autres, elle se propose d'intégrer l'analyse et la compréhension des connaissances, de leur organisation comme de leur application dans une approche du système relationnel interactif liant les humains aux non-humains, et ce, en reconnaissant aux êtres vivants le statut d'être relationnel réservé jusqu'à ce jour aux seuls humains, c'est-à-dire d'être actant et donc interagissant ${ }^{4}$ sur l'élaboration des savoirs. S'assimilant ainsi à une ethnographie de la façon dont les individus perçoivent et conçoivent, dans le cours de leurs interactions avec eux, les comportements des êtres vivants et la manière dont ils réagissent à ces comportements, l'ethnoéthologie cherchera à évaluer, non seulement en quoi les comportements des non-humains - et les interactions qu'ils sous-tendent-, influent sur les savoirs et savoir-faire humains et, au-delà, sur leurs comportements, leur imaginaire et leur conception du monde, mais aussi et réciproquement, en quoi la conception culturelle de ces

3. La publication d'une nouvelle revue internationale intitulée Nature Methode consacrée aux questions méthodologiques indique, s'il le fallait, son enjeu scientifique.

4. Ce que nous invitent à faire l'éthologie et la psychologie comparée qui nous présentent une vision de l'animal complètement différente. Elles lui reconnaissent aujourd'hui des comportements beaucoup moins stéréotypés qu'on ne les considérait jusqu'alors, des capacités cognitives importantes, des variabilités individuelles et même une certaine dimension culturelle. Bref, d'objet quasiment passif et muet, l'animal est devenu « sujet » actant, capable d'intentions et doté d'un savoir non trivial sur le monde et sur les autres (Whiten et al., 1999 ; De Waal, 2001 ; Lestel, 2001). 
individus est susceptible d'influer sur la perception de ces mêmes comportements. Cette ambition scientifique me conduit à apporter quelques précisions sur la méthodologie à développer que j'illustrerai par des exemples issus de mon expérience ethnographique auprès des Kasua.

\section{Appréhender le savoir des autres en reconnaissant qu'ils sont autres}

Prônant le relativisme ontologique comme précepte, l'approche ethno-éthologique doit respecter dans son développement deux principes fondamentaux. Tout d'abord, elle doit s'appliquer a priori à l'ensemble des êtres participant comme définissant le monde dans lequel évolue la société étudiée. Elle devrait donc s'étendre à la faune - à qui nous prêtons volontiers des comportements -, mais également aux plantes, aux minéraux, aux étoiles, voire même aux esprits ou autres manifestations de l'imaginaire. Aussi étonnante puisse-t-elle paraître, cette extension se justifie si l'on considère la conception de la biodiversité des Kasua. Pour ces individus, celle-ci ne se comprend pas dans sa définition biologique mais bien dans sa traduction littérale, c'est-à-dire "la diversité des formes de vies », puisqu'y sont inclus des êtres spirituels actant de façon concrète dans le quotidien forestier (Brunois, 2004). En effet, tout en étant des projections mentales dans le cosmos, ces esprits n'en sont pas moins, pour les Kasua, aussi présents et agissants que le sont les autres formes de vie, c'est-à-dire les humains, les animaux et les plantes. D'ailleurs, chaque esprit se caractérise par des comportements qui lui sont spécifiques et il en est de même des plantes. L'odeur que dégage tel arbre, le fait que le figuier soit étrangleur ou que telle liane soit rampante, épineuse, ou encore à crampons, ne relèvent pas pour les Kasua d'un caractère morphologique botanique, mais bien d'un comportement "personnel à la plante» (Brunois, 2002, 2003) comparable à celui qu'ils reconnaissent aux autres formes de vies qu'ils considèrent comme des alter ego (Brunois, 2001, à paraître).

Enfin, le second principe à respecter dans le cadre d'une ethno-éthologie consisterait à mener notre étude en présence de l'être "vivant». L'intérêt de respecter cette condition de l'enquête réside bien sûr dans l'opportunité qu'elle offre de restituer l'interactivité, nous garantissant la prise en considération du/des comportement(s) agissant(s) de l'être vivant et de l'observateur humain et d'isoler ainsi les divers processus gouvernant la reconnaissance, la nomination comme la catégorisation de l'être rencontré, et au delà, les discontinuités qu'établissent éventuellement les populations entre ces êtres.

\section{L'appréhension, la nomination, la catégortisation au cour de l'interactivité}

Selon le contexte ontologique, le lieu (familier, éloigné, obscur, etc.) et le caractère de la rencontre (fortuit ou volontaire et donc différent selon les intentions), selon l'identité de l'humain (sexe, age, statut, etc.) et celle de l'être vivant, selon leurs comportements respectifs enfin, les sens, les émotions, les réactions qui s'ensuivent chez l'observateur humain peuvent varier d'un individu à un autre, d'une société à une autre. L'examen des ses attitudes est donc important pour préciser les divers facteurs influant les modes d'appréhension, lesquels constituent un préalable à la nomination et à la catégorisation des êtres vivants. Un exemple kasua illustrera le bien-fondé de cette interrogation préliminaire. Les Kasua évoluent dans un cosmos forestier où coexistent des esprits avec qui ils sont dans une relation de réciprocité de vie et de mort. Or, ces esprits peuvent se métamorphoser de manière opportuniste en diverses espèces animales, ce qui confère à la réalité forestière une certaine ambivalence (Brunois, 2001 et à paraître). Le Kasua n'est jamais sûr de l'identité de l'être qui lui fait face. C'est pourquoi, rencontrer par hasard et de jour un animal sur son chemin est suspect à leurs yeux, d'autant que la grande majorité des animaux sont nocturnes. Aussi, pour prévenir un malencontreux malentendu, l'individu va immédiatement se retourner, interpeller l'animal : «si tu es un esprit, va t-en!», puis lui faire face à nouveau et constater sa fuite... Cette réaction est typique et donc récursive. Elle est aussi des plus instructive puisqu'elle nous révèle l'incidence extraordinaire qu'exerce l'ambivalence ontologique sur les modes d'appréhension kasua ${ }^{5}$. Une information capitale donc, qu'une étude menée

5. L'expérience kasua vient ainsi enrichir le débat concernant la théorie essentialiste, à l'instar des Zafimaniry étudiés par Maurice Bloch. Acceptant comme postulats de base, d'une part, la proposition de Scott Atran selon laquelle l'être humain a des dispositions naturelles à reconnaître des domaines spécifiques (1998), d'autre part, que l'intégration systématique des connaissances intervient au dernier stade du développement cognitif (Fentriss, 1984) et, finalement, que la société Zafimaniry n'exprime pas une conceptualisation du monde si dichotomique, Maurice Bloch conclut que ces concepts innés sont susceptibles de se transformer au court du développement cognitif et de perdre ainsi leur caractère prototype et qu'il nous faut dès lors 
hors contexte n'aurait pas pu, bien évidemment, nous communiquer.

Les espaces de «conversation» entre l'homme et son environnement sont également propices à une meilleure compréhension des logiques gouvernant les processus de nomination. Mon enquête auprès des Kasua a révélé en effet combien ceux-ci ne procèdent pas d'une relation abstraite et unilatérale de l'homme au monde, mais d'une expérience interactive ${ }^{6}$. Cette contextualisation est d'autant plus nécessaire que la nomenclature de l'ensemble des êtres, visibles et invisibles, se caractérise par une formidable polysémie: le terme désignant une plante pouvant être partagé par un humain, un animal ou un esprit et vice-versa. Ainsi en est-il, par exemple, de l'essence arborescente Instia bijuga qui présente une morphologie assez singulière au sens où son aubier se teinte d'une couleur rouge sanguine très vive. $\mathrm{Ce}$ critère physiologique contribue incontestablement à son identification. Cependant, sa reconnaissance nécessite d'encocher l'arbre au couteau ou à la hache et son bois est excessivement dur. Aussi, l'enfant apprend à reconnaître l'essence en observant un autre critère plus accessible : la présence dans sa cime du paradisier de Raggi (Paradisae raggiana) qui en a fait sa niche écologique privilégiée. Observant cette relation interspécifique liant ces deux espèces, il pourra alors et sereinement nommer l'arbre par son générique mata qu'il partage avec l'oiseau. La dénomination des batraciens est également riche d'enseignements. Les Kasua pratiquent à leur égard la chasse nocturne à la torche. La technique est très efficace : éblouis, les animaux se paralysent et il suffit de les prendre à la main. Cependant, l'obscurité rend difficile la distinction entre grenouilles comestibles et vénéneuses. Aussi, l'enfant apprend à les identifier en distinguant leur coassement, car «les grenouilles », leur enseigne-t-on, " coassent leur nom ». Ce dernier exemple illustre de nouveau les vertus d'une étude contextualisée. Un inventaire de type naturaliste se contentant de relever les termes désignant les animaux ne nous aurait jamais enseigné sur la diversité des facteurs présidant à la nomination, ni davantage sur cette conception singulière des Kasua qui prête aux autres une auto-désignation, c'est-àdire une autonomie comportementale comme une expressivité. Ce qui traduit bien sûr le fait qu'ils considèrent tous les êtres forestiers comme des êtres relationnels et non des objets. Le traitement taxonomique kasua témoigne parfaitement de cette conception singulière. Leur nomenclature couvre plus d'un millier de taxons (plantes et animaux confondus) formés par un monoléxème et correspond à quelques exceptions près - elles concernent les oiseaux de paradis et le Couscous spilocouscous dimorphiques au niveau scécifique de la reconnaissance scientifique. Elle n'institue donc aucune hiérarchie, conférant à sa base un caractère fort dense. Cette appréhension taxonomique repose sur un ultime critère d'identification généralisé à l'ensemble des composants du cosmos forestier, celui de la niche écologique, soit les relations écologiques qu'entretient chaque espèce avec le reste du monde. L'insertion d'un être vivant dans une catégorie spécifique est en effet soumise à sa reconnaissance, qui selon les Kasua, permet seule d'identifier finalement la "vraie » nature d'animal, de plante, d'humain ou d'esprit. Ce critère opérant exige donc la reconnaissance et la connaissance d'un grand nombre de comportements spécifiques et interspécifiques propres et prêtées à chaque specième afin d'isoler l'éventuel comportement atypique susceptible de trahir l'identité spirituelle de l'être rencontré. Les critères morphologiques ne sont donc d'aucun secours puisqu'ils témoignent d'une apparence qui, pour les Kasua, est par trop ambivalente. Non qu'ils ne connaissent pas parfaitement la physionomie et la physiologie des êtres vivants mais ces connaissances ou ces critères seraient de fait hors contexte ou plutôt limités au contexte présidant à la découpe de l'animal ou à la coupe de l'arbre ! Ce qui explique que mener auprès des Kasua une étude taxonomique objectivée consisterait à chercher « une universalité artificielle de l'ordonnancement» (Descola, 1996: 93). De surcroît, décontextualisée de l'interactivité où elle se pratique, elle nous ôterait l'opportunité d'étudier les différents shèmes présidant aux divers regroupements dont le même être vivant peut faire l'objet ${ }^{7}$, ainsi que les processus

s'interroger et analyser comment d'autres liens se créent et se développent entre ces domaines, initialement, séparés (Bloch, 1998).

6. L'analyse des nomenclatures et des processus de nomination réclame également de se prêter à une nouvelle approche, nommée « translinguistique » dans la mesure où chaque mot serait le résultat cumulatif d'expériences relationnelles interactives entre l'interlocuteur et la communauté parlante (Bakhtine, 1981), et entre l'interlocuteur et les êtres de l'environnement (Basso, 1988 ; Dwyer et Minnegal, 2003)

7. Car, comme le précisait déjà Harold C. Conklin, «à la différence des taxas scientifiques, les ségrégats indigènes peuvent appartenir à plusieurs structures hiérarchiques distinctes » (1962:119). 
d'identification appliqués aux êtres vivants ${ }^{8}$. Or, mener in situ, cette analyse permettrait d'expliquer les critères opérant les discontinuités entre les espèces et les différents « règnes » auxquels elles appartiennent, mais aussi d'isoler l'interférence des conceptions ontologiques dans la reconnaissance de ces discontinuités. Chez les Kasua, les critères morphologiques ont une efficience différentielle limitée. D'ailleurs, c'est un même vocabulaire qui s'applique indifféremment à la description de la physiologie des êtres quelle que soit leur catégorie d'appartenance. Ainsi, le corps des humains, des arbres ou des animaux est parcouru par les mêmes mots « substanciels »: hano, l'eau, la sève ; $m a$, la résine, la syprine ; $i b i$, la graisse fertile, le latex, parfois nommé secrètement kole, c'est-à-dire sperme (Brunois, 2003). Il en va de même du vocabulaire décrivant les comportements observés par l'ensemble des formes de vies. Et pourtant, ces derniers sont aussi divers que variés. Les Kasua identifient ainsi l'expressivité comme mode de stratégie de différenciation, la communication spécifique et interspécifique, l'intelligence et la conscience réflexive, toutes les formes de sensibilité, la capacité mimétique, l'hétérosexualité et l'homosexualité pratiquée par les deux sexes, les stratégies de séduction et d'accouplement, les sentiments, les émotions, la paresse, la curiosité, la sournoiserie, l'humour, la monogamie et la polyandrie, la fidélité et l'adultère, la recherche de l'esthétisme, la lenteur et la vitesse, le comportement nocturne ou diurne, la capacité de rêver, les différents modes d'alimentation (carnivore, frugivore, insectivore, omnivore), les stratégies alimentaires (l'exo-cannibalisme, la prédation opportuniste, le vol, la ruse comme l'imitation des cris de la proie ou la connaissance de ses habitudes, la collecte, la cueillette, le jardinage), les stratégies de défense (bataille, mimétisme, camouflage, l'aménagement d'une sortie de secours, fuite), la capacité de bâtir et, enfin, la transmission des savoirs... La liste est ici suffisamment longue pour témoigner de la sensibilité éthologique des Kasua, laquelle, nous l'avons $\mathrm{vu}$, joue un rôle essentiel pour la reconnaissance, la nomination et la catégorisation des êtres vivants. Cependant, cet inventaire peut paraître troublant tant nombre de ces comportements relève communément de l'humanité. Est-ce à dire que les processus d'identifications observés par ces individus reposent sur de l'anthropomorphisme ou encore du métaphorisme ? Une étude hors contexte aurait sans doute conduit à cette conclusion. Celle-ci, il est vrai, ne contredirait pas le fait que ces analogies comportementales trahissent la singularité de l'ontologie kasua puisqu'elle n'établit aucune discontinuité entre les différentes formes de vies. En revanche, elle réduirait formidablement la complexité des processus gouvernant l'identification des Kasua aux êtres « vivants ». Comme je l'ai montré ailleurs (Brunois, sous presse), ceux-ci reposent en effet sur une empathie que j'ai qualifié d'interspécifique, soit la capacité des Kasua, sans confusion entre eux même et les autres, à se mettre à leur place pour ressentir leur "Umwelt» (Uexküll, 1982). Bien sûr, cette compréhension empathique de l'autre recourt à la pensée analogique, mais celle-ci n'agit pas seule et a priori. Si les représentations kasua favorisent certainement une identification positive aux autres, elle ne prêtent aux êtres « vivants », ni des comportements universels, ni une sociabilité humaine (à l'exception des esprits), ni davantage une intentionnalité généralisée à tous. Les analogies que reconnaissent les Kasua et qui relèvent, nous l'avons vu, du domaine des techniques autant que du domaine de la sexualité ou encore de celui de l'expressivité, résultent d'un raisonnement s'enrichissant de leurs observations quotidiennes du comportement des êtres et de leurs expériences partagées et renouvelées avec eux. Autrement dit, leurs processus d'identification associent le savoir, la phénoménologie, la perception, les émotions et la prise de perspective sur l'autre, c'est-à-dire l'intersubjectivité témoignant d'une ontologie soucieuse du caractère interactif des relations liant les homme aux non-humains.

En somme, accepter avec les Kasua le caractère mutuel des rapports homme/environnement signifie certes reconnaître les comportements spécifiques et écologiques des êtres vivants, mais cela signifie aussi admettre leur faculté à interagir sur le comportement des humains. Ce qui m'amène à conclure sur un dernier point fondamental, celui de l'incidence qu'exerce le mode d'existence des êtres vivants sur les savoir-faire écologiques.

\section{L'incidence des comportements des êtres vivants sur les savoir-faire écologiques}

$\mathrm{Si}$, jusqu'à présent, je me suis consacrée à démontrer la pertinence d'aborder les modes de connaissances - leur élaboration comme leur

8. En s'interrogeant sur leurs modalités respectives : quels vocabulaires et expressions linguistiques leurs sont associés ? Relèvent-ils de l'anthropomorphisme, du métaphorisme, du métonymisme, du « spirimorphisme », du matérialisme, ou encore du phénoménologique? 
organisation - en empruntant une perspective interactive, je voudrais poursuivre cette démonstration en mettant en lumière l'intérêt d'étendre cette approche à la compréhension des savoirfaire. En effet, si nous voulons isoler tous les facteurs présidant à la formation des savoir-faire écologiques, nous sommes tenus là encore de prendre en considération les relations spécifiques et interspécifiques qu'instituent les êtres vivants. Quelques exemples illustreront mon propos. Si un Kasua veut chasser un Spilocuscus tacheté, un marsupial arboricole à l'attitude nonchalante et aux yeux aussi globuleux qu'attendrissants, il évitera à tout prix de croiser son regard qui le ferait pleurer et l'empêcherait de décocher sa flèche. La stratégie cynégétique consiste donc, et toujours, à tirer la flèche d'une distance suffisamment éloignée. De même, lorsqu'un individu rencontre le martin-pêcheur Ceyx lepidus, reconnu pour participer d'une relation de commensalisme avec l'anguille, il renoncera à le flécher, préférant le préserver et suivre son vol jusqu'aux rivières en espérant capturer le poisson. Un bûcheron ouvrant un essart évitera de couper certaines essences arborescentes car elles entretiennent une relation symbiotique avec des animaux ou encore des esprits. Il en sera ainsi avec plus de quatre-vingt-dix espèces d'arbres dont la regénérescence est assurée par le casoar, ce qui conduit les Kasua à affirmer volontiers qu'il «est l'horticulteur de la forêt » (Brunois, 2001 et à paraître). L'incidence qu'exerce les modes d'existene des êtres vivants sur les pratiques kasua est ici évidente. Ces exemples, choisis parmi tant d'autres, nous révèlent tout d'abord combien la reconnaissance de ces comportements et des relations qu'ils sous-tendent entre les différents êtres (dont les humains) confère au savoir-faire kasua une portée fondamentalement écologique ${ }^{9}$. Enfin, et de façon tout aussi déterminante, ces exemples nous démontrent combien leur savoir-faire s'en enrichit. D'ailleurs, les Kasua ne le cachent pas. Interrogés sur des homologies que j'avais constatées entre certains comportements observés par les Kasua (technique, alimentaire, cérémoniel ou encore guerrier) avec ceux de ceratins animaux co-évoluant dans leur forêt, ils m'ont répondu tout simplement : « nous avons copié10 ${ }^{10}$ (Brunois, sous presse), donnant ainsi raison sans le savoir à l'intuition
d'André G. Haudricourt. Déplorant qu' « en ce qui concerne notre relation avec les animaux, nous n'en savons guère plus qu'avec les plantes », il souhaitait que l'ethnozoologie «se dégage de la zootechnie où elle est née pour s'intéresser vraiment aux relations réciproques de l'homme et de l'animal. [...] Il faudrait", ajoutait-il, "que les chercheurs se débarrassent d'un certain nombre de tabous qui ne leur permettent pas de "regarder" complètement une société et ainsi de n'en comprendre jamais qu'une partie. L'homme a ses gestes dans la nature et je pense que pour trouver l'explication des différences de comportement humain, il faut se référer aux animaux ${ }^{11}$ qu'il fréquente ou qui l'environnent » (Haudricourt et Dibie, 1987 : 169).

\section{Conclusion}

Regarder complètement le savoir et savoirfaire kasua exige effectivement d'observer complètement les êtres qu'ils fréquentent, plus encore, de re-instaurer l'interactivité entre les Kasua et les êtres vivants et de l'écouter pour mesurer l'impact qu'exercent la présence et l'action de ces êtres sur leurs savoirs et savoirfaire. En d'autres termes, appréhender leurs modes de connaissances en reconnaissant qu'ils sont autres contraint à réviser notre traditionnelle appréhension des savoirs locaux ${ }^{12}$.

Leur expérience nous enseigne que leur approche est en effet plus complexe qu'il n'y paraît au sens où elle n'est pas le fait d'une population exclusivement humaine - les Kasua- mais le résultat d'une expérience interspécifique. Aussi, restituer l'originalité de leurs modes de connaissance réclame de dés-humanocentrer notre milieu de vie, et simultanément, d'accorder aux êtres vivants le statut d'acteurs, puisqu'au même titre que les humains, ils interagissent sur la constitution du savoir. C'est au prix de cette double recontextualisation - écologique et ontologique -, qu'il nous sera possible de rendre compte de la dynamique des savoirs des autres et de nous prêter à des comparaisons des épistèmes hier encore impensables.

9. La forêt des Kasua représente ainsi le théâtre d'une sociabilité subtile où humains et non humains se maintiennent dans un équilibre relationnel délicat, celui-là même que définit l'ontologie kasua (Brunois, 2001, 2004, à paraître).

10. Ce qui explique que mener une étude sur les usages de la biodiversité chez les Kasua exige d'élargir son champ d'enquête. Par exemple, l'utilité des plantes ne peut se comprendre dans son sens traditionnellement entendu, c'est-à-dire, " humanocentrique ». Pour les Kasua en effet, une plante est aussi utile car nécessaire aux esprits ou encore aux animaux cohabitant leur massif forestier. J'ai relevé ainsi 483 usages que tire la faune de la flore herborisée (Brunois, 2002).

11. Et aux plantes (Brunois, 2003)

12. Et au-delà, celle du phénomène culturel (Brunois, à paraître). 


\section{BIBLIOGRAPHIE}

ARHEM Kaj, 1996. The cosmic food web: humannature relatedness in the Northwest Amazon, in Philippe Descola and Gisli Palsson (eds), Nature and Society. Anthropological perspectives, London and New York, Routledge, pp. 185-205.

Atran Scott, 1998. Folkbiology and the anthropology of science: Cognitive universals and cultural particulars, Behavioral and Brain Sciences 21, pp. 547-595.

Bahuchet Serge, 1989. Les noms d'arbres des Pygmées de l'ouest du Bassin congolais, Adeansoia II, 4, pp. $355-365$

BaKhtine Mikhail, 1981. The Dialogic Imagination: Four Essays, Austin, University of Texas Press.

BARRAU Jacques, 1967. De l'homme cueilleur à l'homme cultivateur: l'exemple océanien, Cahiers d'histoire mondiale 10, 2, Neuchâtel, pp. 275-292.

_, 1971. L'ethnobotanique au carrefour des sciences naturelles et des sciences humaines, Bulletin de la Société botanique française 118, pp. 237-248.

—, 1975. Écosystèmes, civilisations et sociétés humaines, le point de vue d'un naturaliste. Information en sciences sociales 14, pp. 21-34.

BAsso Keith, 1988. 'Speaking with names': Language and landscape among the Western Apache. Cultural Anthropology 3, pp. 99-130.

Bloch Maurice, 1998. How We Think They Think, Colorado, Westview Press.

BRUNoIs Florence, 2001. Le jardin du Casoar, la forêt des Kasua. Influences des relations au milieu forestier sur la constitution de l'identité et des savoirs et savoir-faire écologiques de la société kasua, mémoire de doctorat à l'EHESS-Paris, 427 p., +87 p. annexes, $1 \mathrm{CD}$-rom.

—, 2002. Du dessin au dessein des plantes sauvages, Journal de la Société des Océanistes 114-115, pp. 2339.

—, 2003. Être arbre: la condition humaine kasua (Nouvelle-Guinée) visitée par la condition du végétal ou vice-versa, Cahiers de littérature orale 53-54, pp. 293-304.

—, 2004. La forêt peut-elle être plurielle ? Définitions de la forêt des Kasua de Nouvelle-Guinée, Anthropologie et Sociétés 28, 1, pp. 89-107.

_, (sous presse). Man or animal: who copies who? Interspecific empathy and imitation among the Kasua of New Guinea, in Alessandro Minelli, Gherardo Ortalli \& Clauco Sanga (eds), Animal Names, Venise, Instituto Veneto di Scienze, Lettere ed Arti, pp. 389-401.

-, (à paraître). Le jardin du casoar, la forêt des Kasua. Ontologie, savoir et savoir-faire écologiques de la société kasua. Papouasie-Nouvelle-Guinée, Paris, Éditions CNRS-MSH, coll. Chemins de l'Ethnologie.
BULMER Ralph, 1967. Why is the cassowary not a bird? A problem of zoological taxonomy among the Karam of the New Guinea Highlands, Man 2, pp. 5-25.

_, 1968. Worms that Croak and Other Mysteries of Karam Natural History, Mankind 6, pp. 621-639.

Chaumeil Jean-Pierre,1983. Voir, savoir, pouvoir : le chamanisme chez les Yagua du nord-est peruvien, Paris, Éditions de l'École des hautes études en sciences sociales.

ConkLIN Harold,1962. Lexicographical treatment of Folk taxonomies, International Journal of American linguistics, pp. 129.

Descola Philippe, 1986. La nature domestique. Symbolisme et praxis dans l'écologie des Ashuar, Paris, MSH.

—, 1996. Constructing natures: symbolic ecology and social practice, in Philippe Descola and Gisli Palsson (eds), Nature and Society. Anthropological perspectives, London and New York, Routledge, pp. 82103.

Descola Philippe et Gisli Palsson, 1996. Introduction, in Philippe Descola and Gisli Palsson (eds) Nature and Society. Anthropological perspectives, London and New York, Routledge, pp. 1-23.

DWYeR Peter, 1976. Beetles, butterflies and bats: Species transformation in a New Guinea folk classification, Oceania 46, 2, pp. 188-205.

_, 1984-1985. Other people's animals: Two examples from New Guinea, Search 15, 11-12, pp. 321-327.

-, 1996. The Invention of Nature, in Roy ElLEN and Kiyoshi FukuI (eds), Redefining Nature: Ecology, Culture an Domestication, Oxford, Berg, pp. 157186.

Dwyer Peter, Roger Just et Monica Minnegal, 2003. A sea of small names: Fishers and their boats in Victoria, Australia. Anthropological Forum 13, 1, pp. 5-26.

ElLEN Roy, 1993. The Cultural Relations of Classification. An Analysis of Nuaulu Animal Categories from Central Seram. Cambridge: Cambridge University Press.

ElLEN Roy et Kiyoshi FukuI (eds), 1996. Redefining Nature: Ecology, Culture and Domestication, Oxford, Berg.

Fentriss J. C., 1984. The development of coordination, Journal of Motor Behaviour 16, pp. 99-134.

FrIEDBERG Claudine,1990. Le savoir botanique des Bunaq. Percevoir et classer dans le haut Lamaknen, Paris, Mémoires du Muséum national d'histoire naturelle, Botanique 32.

—, 1997. Diversité, ordre et unité du vivant dans les savoirs populaires, Nature, science et société 5, 1, pp. 5-17.

Gibson James, 1979. The Ecological Approach to Visual Perception, Boston, Houghton Mifflin.

Goody Esther, 1978, Towards a Theory of Questions, in Goody (ed), Questions and Politeness: strategies 
in social interaction, Cambridge, Cambridge University Press

Grenand Pierre, 1980. Introduction à l'étude de l'univers wayapi: ethno-écologie des indiens du hautOyapock (Guyane française), Paris, SELAF/CNRS.

HAudricourt André Georges, 1962. Domestication des animaux, cultures des plantes et traitement d'autrui, L'Homme II, pp. 40-50.

-, 1964. Nature et culture dans la civilisation de l'igname: l'origine des clones et des clans. L'Homme IV, pp. 93-104.

Haudricourt André Georges et Pascal Dibie, 1987. Les pieds sur terre, Paris, A. M. Metaillé.

HornBorg Alf, 1996. Ecology as semiotics: outlines of a contextualist paradigm for human ecology, in Philippe Descola and Gisli Palsson (eds), Nature and Society. Anthropological perspectives London and New York, Routledge, pp. 45-63.

Howell Signe, 1996. Nature in culture or culture in nature? Chewong ideas of "humans and other species, in Philippe Descola and Gisli Palsson (eds), Nature and Society. Anthropological perspectives, London and New York, Routledge, pp. 127145.

HvidING Edvard, 1996. Nature, culture, magic, science: on meta-languages for comparison in cultural ecology, in Philippe Descola and Gisli Palsson (eds), Nature and Society. Anthropological perspectives, London and New York, Routledge, pp. 165185.

INGOLD Tim, 1996. Hunting and Gathering as Ways of Perceiving the Environment, in Roy Ellen and Kiyoshi Fukui (eds), Redefining Nature: Ecology, Culture an Domestication, Oxford, Berg, pp. 117154.

Latour Bruno, 1979. Laboratory Life, Newsbury Park, Sage.
—, 1991. Nous n'avons jamais été modernes. Essai d'anthropologie symétrique, Paris, La Découverte.

LAVE Jean, 1993. The Practice of Learning, in Seth Chaiklin et Jean Lave (eds). Understanding Practice: Perspectives on Activity and Contex, Cambridge, Cambridge University Press .

Lestel Dominique, 2001. Les origines animales de la culture, Paris, Flammarion.

Maturana Humberto et Francesco Varela, 1987. The trees of Knowledge: The Biological Roots of Human Understanding, Boston, Shambhala.

Minnegal Monica, 1996. A necessary unity: the articulation of social and ecological explanations of behaviour, Journal of the Royal Anthropological Institute n.s. 2, pp. 141-58.

Nothnagel Detlev, 1993. Anthropologische Feldforschung in Organisationen, Anthropos 88, pp. 459476.

ReVel Nicole, 1990. Fleurs de Paroles. Histoire naturelle Palawan. Tome 1. Les dons de Nägsalad, Paris, Éditions Peeters-SELAF 314.

STENGERS Isabelle, 1995. L'Invention des sciences modernes, Paris, Champs Flammarion.

STRATHERn Marylin, 1980. No Nature, No Culture, in Carol MacCormack et Marylin Strathern (eds), Nature, Culture and Gender, Cambridge, Cambridge University Press, pp. 180-220.

Viveiros de Castro Eduardo, 1996. Cosmological Deixis and Amerindian Perspectivism, Journal Roy. anthrop. Inst 4, pp. 469-488.

Von UexküLL Jakob, 1982 (1940). The theory of meaning, Semiotica 42, pp. 25-82.

WAAL Frank (de), 2001. Quand les singes prennent le thé. De la culture animale. Paris :Fayard.

WHITEN Andrew et al., 1999. Cultures in chimpanzees, Nature 399, pp. 682-685. 